\title{
"Low back pain response after application of interferential therapy alone and in combination with aceclofenac + paracetamol, tramadol+paracetamol: A prospective, comparative, clinical study"
}

Surendra Kumar Vidyarthi, Roopa Prasad Nayak, Satish Krishna, Prakash Selvaraj, Sandeep Kumar Gupta, Balasubramanian Anantha Kumar

Department of Pharmacology, Dhanalakshmi Srinivasan Medical College and Hospital (DSMCH), Siruvachur, Perambalur, Tamil Nadu, India

Address for the Correspondence: Dr. Surendra Kumar Vidyarthi, Department of Pharmacology, Dhanalakshmi Srinivasan Medical College and Hospital (DSMCH), Siruvachur - 621113 , Perambalur, Tamil Nadu, India E-mail: skvmanju9208@ yahoo.co.in

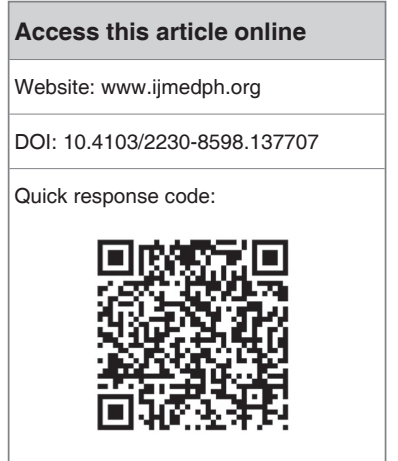

Aims and Objectives: To determine efficacy of analgesia of Analgesic drugs + Interferential Therapy (IFT) or without IFT. Material and Methods: The 80 eligible patients ( 30 male, 50 female) of acute and chronic low back pain were assigned to 5 groups (Grp), i.e., Grp A, B, C, D and E. The Orthopedician prescribed tablet Xenodol (Aceclofenac 100mg + Paracetamol 500mg 1 BD for 7 days) for Grp A, tablet Patrol (Tramadol 37.5mg + Paracetamol 325mg) 1 BD for 7 days) for Grp B, IFT $25-100 \mathrm{~Hz}$ application at lumbar region for 15 min daily for 7 days for Grp C, tablet Xenodol of same dose with IFT $25-100 \mathrm{~Hz}$ application for 7 days for Grp D and tablet Patrol of same dose with IFT $25-100 \mathrm{~Hz}$ application for 7 days for Grp E patients. Eligible patients pain were assessed twice, i.e., pre-treatment and post-treatment, by SF-LF-MPQ (Short Form-Long Form McGill Pain Questionnaire's Rating Index. Results: The post-treatment mean $\pm S D$ was $1.3 \pm 0.9,1.4 \pm 1.5,1.6 \pm 0.85,1 \pm 0.73,0.93 \pm 1.16$ for Grp A, B, $C, D$ and $E$ respectively, while the pre-treatment pain intensity score mean $\pm S D$ was $1.9 \pm 0.5,2.4 \pm 1.1,2.13 \pm 0.8,2.25 \pm 0.9,2.25 \pm 1.1$ for Grp A,B,C,D,E respectively. The differences was statistically significant, i.e. $(P<0.05) 0.03856,0.03601$, $0.0002027,0.002136$ of Grp A, B, D, E respectively and insignificant, i.e. $(P>0.05)$ was 0.06603 for Grp C. Conclusion: Tablet Xenodol (Aceclofenac + Paracetamol) and tablet Patrol (Tramadol + Paracetamol)alone showed efficacy in pain reduction, while when the tablets Xenodol and tablet Patrol of same doses given with IFT $25-100 \mathrm{~Hz}$ application at lumbar regionin Grp D and Grp $E$ the pain reduction was highly significant than the medication alone groups, i.e., for Grp A, B, C.

Key words: Aceclofenac+paracetamol, interferential therapy (IFT ), low-back pain (LBP ), McGill pain questionnaires, tramadol+paracetamol

\section{INTRODUCTION}

Low Back Pain is highest prevalence in the aged 45 to 65 years. ${ }^{[1]}$ The 60 to $80 \%$ world population experienced LBP. ${ }^{[2]}$ There is multiple non-surgical LBP treatment modalities available like: e.g. Counseling and education, rest, medication, braces, passive modalities, spinal manipulation, injection, exercise and stretching, proper lifting technique. Acetaminophen and other non-steroidal antiinflammatory drugs are commonly used for the treatment of LBP. ${ }^{[3]}$

Electrical stimulation has widespread clinical use for pain relief. The $90-130 \mathrm{~Hz}$ frequencies stimulate the pain gate mechanisms and mask the pain symptoms, while stimulation with lower frequencies $(2-5 \mathrm{~Hz})$ can be used to activate the opioid mechanisms to providing a degree of pain relief. It is possibility that relief of pain may be achieved by stimulation of the reticular formation at frequencies of $10-25 \mathrm{~Hz}$ or by blocking $\mathrm{C}$ fiber transmission at $>50 \mathrm{~Hz}$. Thus, Electrotherapy (Electro Physical Agents) has been possible used in clinical practice appropriately to relief pain, the evidence supports its effectiveness. ${ }^{[4]}$

So, IFT W-30, Range 1 to $250 \mathrm{~Hz}$, manufactured by 'HMS Medical System' Chennai, electrical stimulator used in present study for comparison of analgesic efficacy of IFT $(25-100 \mathrm{~Hz})+$ tab. 
Xenodol (Aceclofenac+Paracetamol), IFT (25-100 Hz) + tab. Patrol (Tramadol + Paracetamol) and IFT $(25-100 \mathrm{~Hz})$ alone in another group for the present study.

\section{MATERIALS AND METHODS}

After approval of the present study from the Institutional Clinical Ethics Committee, total 80 assigned patients $(30$ male and 50 female) segregated in five groups by using lottery method and prescribed the study therapy [Table 1]. The study was prospective, clinical study. The duration of the study was 7 days dated from $2^{\text {nd }}$ July 2013 to $9^{\text {th }}$ July 2013 with 7 days follow-up. Study Centre was: Out-patient Orthopedics and Physiotherapy and Pharmacology Department, DSMCH (Dhanalakshmi Srinivasan Medical College and Hospital, Perambalur, Tamilnadu, India. Sample Selection Criteria: Inclusion Criteria: The patients, who complained acute or chronic low-back pain, No history of taking analgesics in the previous one month and male and female of ages between 30 to 60 years with LBP, were included in the study. Exclusion Criteria: If any patient does not come under inclusion criteria, post-operative patients, patients who suffered from peptic ulcers, congestive heart failure, liver and renal impairment, multiple injuries with bony fractured patients, pain due to any cause; except related to acute or chronic low-back pain, unwilling to participate in the study patients; excluded from the study. If patients absconded from the study excluded too but, that shown as dropout in percent $(\%)$ during calculation.

The study drugs provided from the DSMCH Pharmacy and Department of Physiotherapy applied IFT 25-100 Hz for $15 \mathrm{~min}$ daily over lumbar region in Grp C, D and in Grp E patients for seven days. The IFT specification used for the study was W-30, Range 1-250 Hz, manufactured by 'HMS Medical System' Chennai, electrical stimulator. We assessed pain intensities grade, i.e., (0...No Pain, 1....Mild, 2....Discomforting, 3.....Distressing, 4...... Horrible, 5.....Excruciating)by Short Form-Long Form McGill Pain Questionnaire $^{[5]}$ (SF-LF-MPQ). We noted the Pain intensities twice, first (Baseline pain/pre-treatment), i.e., at the time of registration of the patients and second (post-treatment), after end of seven days of the therapy.

\section{Statistical calculation}

After obtaining the data, we calculated the values, like percent, Mean, Standard Error, Standard Deviation, Dropout Rate from the study (in percent) and $P$-value by applying various statistical formulas, like' paired t-test', Kruskalwallis test (ANOVA) through online/offline free software, i.e., www.openepi.com.

\section{RESULTS}

The baseline pain intensity (mean) was 1.9, 2.44, 2.13, 2.25, 2.25 for Grp A, B, C, D, E treated patients respectively and post-treatment was 1.3, 1.4, 1.57,1, 0.93 of Grp A, B, C, D, E treated patients respectively [Tables 2,3 and Figure 1]. Thus, after $7^{\text {th }}$ day of medication, pain reduction mean was 39.3, 53.3, 23.2, 61.9, 61.7\% of Grp A, B, C, D, E patients respectively [Tables 2,3 and Figure 2].

After 7 days, i.e., post-treatment, the 4 patients had $100 \%$ and 3 patients had $50 \%$ pain relief of Grp A, the 7 had $100 \%$ and 2 had

\begin{tabular}{lllll} 
Table 1: Therapy for the Study Groups & & & \\
\hline Grp A & Grp B & Grp C & Grp D & Grp E \\
\hline Tablet Xenodol (Aceclofenac & Tablet Patrol (Tramadol & IFT 25-100 Hz Electrical & Tablet Xenodol 1 BD & Tablet Patrol 1BD + \\
$100 \mathrm{mg}+$ Paracetamol & $37.5 \mathrm{mg}+$ Paracetamol 325 mg) & Stimulator application, & + IFT Application & IFT Application daily \\
$500 \mathrm{mg}$ ) 1 BD daily for & 1 BD daily for 7 days & 15 min, at Lumbar region & daily at lumbar region & at lumbar region for \\
7 days & & daily for 7 days & for 7 days & 7 days \\
\hline
\end{tabular}

\begin{tabular}{|c|c|c|c|c|c|c|c|c|c|c|}
\hline Groups & Therapy given & $\begin{array}{l}1^{\text {st }} \text { day pain } \\
\text { intensity } \\
\text { (Mean) }\end{array}$ & $\begin{array}{l}7^{\text {th }} \text { day } \\
\text { pain } \\
\text { intensity } \\
\text { (Mean) }\end{array}$ & $\begin{array}{l}1^{\text {st }} \text { day } \\
\text { pain } \\
\text { intensity } \\
\text { (SD) }\end{array}$ & $\begin{array}{l}7^{\text {th }} \text { day } \\
\text { pain } \\
\text { intensity } \\
\text { (SD) }\end{array}$ & $\begin{array}{l}1^{\text {st }} \text { day } \\
\text { pain } \\
\text { intensity } \\
\text { (SE) }\end{array}$ & $\begin{array}{l}7^{\text {th }} \text { day } \\
\text { pain } \\
\text { intensity } \\
\text { (SE) }\end{array}$ & $\begin{array}{l}1^{\text {st }} \text { day } \\
\text { pain } \\
\text { intensity } \\
(\%)\end{array}$ & $\begin{array}{l}7^{\text {th }} \text { day } \\
\text { pain } \\
\text { intensity } \\
\text { reduction } \\
(\%)\end{array}$ & $\begin{array}{l}\text { Dropout } \\
\text { rate (\%) }\end{array}$ \\
\hline Grp A & $\begin{array}{l}\text { Tablet Xenodol (Aceclofenac } \\
100 \mathrm{mg}+\text { Paracetamol } 500 \mathrm{mg} \text { ) } \\
\text { 1BD daily for } 7 \text { days }\end{array}$ & 1.9 & 1.3 & 0.5 & 0.9 & 0.13 & 0.25 & 100 & 39.3 & 12.50 \\
\hline Grp B & $\begin{array}{l}\text { Tablet Patrol (Tramadol } \\
37.5 \mathrm{mg}+\text { Paracetamol } 325 \mathrm{mg} \text { ) } \\
1 \text { BD daily for } 7 \text { days }\end{array}$ & 2.4 & 1.4 & 1.1 & 1.5 & 0.3 & 0.4 & 100 & 53.3 & 6.25 \\
\hline Grp C & $\begin{array}{l}\text { IFT } 25-100 \mathrm{~Hz} \text { Electrical Stimulator } \\
\text { application, } 15 \text { minutes, at Lumbar } \\
\text { region daily for } 7 \text { days }\end{array}$ & 2.13 & 1.6 & 0.81 & 0.85 & 0.20 & 0.21 & 100 & 23.2 & 12.50 \\
\hline Grp D & $\begin{array}{l}\text { Tablet Xenodol 1BD + IFT } \\
\text { Application daily for } 7 \text { days }\end{array}$ & 2.25 & 1 & 0.93 & 0.73 & 0.5 & 0.25 & 100 & 61.9 & 12.50 \\
\hline Grp E & $\begin{array}{l}\text { Tablet Patrol 1BD + IFT } \\
\text { Application daily for } 7 \text { days }\end{array}$ & 2.25 & 0.93 & 1.1 & 1.16 & 0.27 & 0.29 & 100 & 61.7 & 6.25 \\
\hline
\end{tabular}


$50 \%$ pain reduction of Grp B, the 1, 4, 1 patients reported $100 \%$, $50 \%, 25 \%$ pain reduction respectively of Grp C, the $4,2,6,1$, patients had $100 \%, 66.7 \%, 50 \%, 33.3 \%$, pain reduction respectively of Grp D, the 7, 4, 1, patients pain reduction was $100 \%, 50 \%, 25 \%$ respectively of Grp E [Table 3 and Figure 3].

The no reduction of pain intensity after 7 days of medication of Grp A, B, C, D, E was 7, 6, 8, 1, 3 patients respectively [Table 4].The 2, 1, 2, 2, 1 patients,(i.e., dropout rate $12.50,6.25,12.50,12.50,6.25 \%$ ) had not reported (reason unknown) after 7 days of the medication of Grp A, B, C, D, E respectively [Table 3 and Figure 4].

The Statistical analysis done by applying paired t-tests and Kruskalwallis test (ANOVA) through using Online/offline Free Software, eg.www.openepi.com .Between the groups Pain intensities was reduced highly significantly $(P=0.00001)$ [Table 4].

The differences of pain reduction was statistically significant, i.e. $(P<0.05)$ 0.03856, 0.03601, 0.0002027, 0.002136 of Grp A, B, D,

\begin{tabular}{|c|c|c|c|c|c|c|c|c|c|c|c|}
\hline \multirow[t]{2}{*}{ Groups } & \multirow{2}{*}{$\begin{array}{c}\text { Age year Mean } \\
\text { and (SD) of the } \\
\text { patients }\end{array}$} & \multirow[t]{2}{*}{$\begin{array}{l}\text { No. of } \\
\text { Patients }\end{array}$} & \multirow[t]{2}{*}{$\begin{array}{l}\text { No. of } \\
\text { Male }\end{array}$} & \multirow[t]{2}{*}{$\begin{array}{l}\text { No. of } \\
\text { Female }\end{array}$} & \multicolumn{6}{|c|}{$\begin{array}{l}\text { After } 7 \text { th day of medication number of patients get } \\
\text { reduced pain intensity }\end{array}$} & \multirow{2}{*}{$\begin{array}{c}\text { Not } \\
\text { reported } \\
\text { patients }\end{array}$} \\
\hline & & & & & $\downarrow 100(\%)$ & $\downarrow 66.7(\%)$ & $\downarrow 50(\%)$ & $\downarrow 33.3(\%)$ & $\downarrow 25(\%)$ & $\downarrow 0(\%)$ & \\
\hline Grp A & $41.75 \pm 11.67$ (SD) & 16 & 8 & 8 & 4 & - & 3 & - & - & 7 & 2 \\
\hline Grp B & $43.63 \pm 11.77(\mathrm{SD})$ & 16 & 4 & 12 & 7 & - & 2 & - & - & 6 & 1 \\
\hline Grp C & 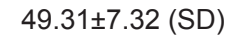 & 16 & 7 & 9 & 1 & - & 4 & - & 1 & 8 & 2 \\
\hline Grp D & $43.25 \pm 7.72(\mathrm{SD})$ & 16 & 6 & 10 & 4 & 2 & 6 & 1 & - & 1 & 2 \\
\hline Grp E & $46.38 \pm 10.99(S D)$ & 16 & 5 & 11 & 7 & - & 4 & - & 1 & 3 & 1 \\
\hline
\end{tabular}

\begin{tabular}{|c|c|c|c|c|c|c|}
\hline & \multicolumn{4}{|c|}{ Input Data } & & \\
\hline & Group & $\mathrm{N}$ (count) & Mean & Std. Dev. & & \\
\hline & GrpA: $1^{\text {st }}$ day & 16 & 1.9 & 0.5 & & \\
\hline & $\operatorname{GrpA}: 7^{\text {th }}$ day & 16 & 1.3 & 0.99 & & \\
\hline & GrpB: $1^{\text {st }}$ day & 16 & 2.44 & 1.09 & & \\
\hline & GrpB: $7^{\text {th }}$ day & 16 & 1.4 & 1.55 & & \\
\hline & GrpC: $1^{\text {st }}$ day & 16 & 2.13 & 0.81 & & \\
\hline & GrpC: $7^{\text {th }}$ day & 16 & 1.57 & 0.85 & & \\
\hline & GrpD: $1^{\text {st }}$ day & 16 & 2.25 & 0.93 & & \\
\hline & GrpD: $7^{\text {th }}$ day & 16 & 1 & 0.73 & & \\
\hline & GrpE: $1^{\text {st }}$ day & 16 & 2.25 & 1.06 & & \\
\hline & GrpE: $: 7^{\text {th }}$ day & 16 & 0.93 & 1.16 & & \\
\hline \multicolumn{7}{|c|}{ ANOVA Table } \\
\hline Source of variation & Sum of squares & & d.f & Mean square & F statistics & $P$-value ${ }^{[1]}$ \\
\hline Between Groups & 43.5906 & & 9 & 4.8434 & 4.8115 & 0.0000119713 \\
\hline Within Groups & 150.995 & & 150 & 1.00663 & & \\
\hline \multirow[t]{2}{*}{ Total } & 194.585 & & 159 & & & \\
\hline & & Chi square & d.f & $P$-value ${ }^{[1]}$ & & \\
\hline \multirow{2}{*}{$\begin{array}{l}\text { Test for equality of } \\
\text { variance }\end{array}$} & & 22.4211 & 9 & 0.0076361 & & \\
\hline & \multicolumn{3}{|c|}{$95 \% \mathrm{Cl}$ of individual sample mean } & & \multicolumn{2}{|c|}{$95 \% \mathrm{Cl}$ assuming equal variance } \\
\hline Group & Mean & Lower limit & Upper limit & & Lower limit & Upper limit \\
\hline GrpA: $1^{\text {st }}$ day & 1.9 & 1.63357 & 2.16643 & & 1.36538 & 2.43462 \\
\hline $\operatorname{GrpA}: 7^{\text {th }}$ day & 1.3 & 0.772468 & 1.82753 & & 0.765376 & 1.83462 \\
\hline GrpB: $1^{\text {st }}$ day & 2.44 & 1.85918 & 3.02082 & & 1.90538 & 2.97462 \\
\hline GrpB: $7^{\text {th }}$ day & 1.4 & 0.574066 & 2.22593 & & 0.865376 & 1.93462 \\
\hline GrpC: $1^{\text {st }}$ day & 2.13 & 1.69838 & 2.56162 & & 1.59538 & 2.66462 \\
\hline GrpC: $7^{\text {th }}$ day & 1.57 & 1.11707 & 2.02293 & & 1.03538 & 2.10462 \\
\hline GrpD: $1^{\text {st }}$ day & 2.25 & 1.75444 & 2.74556 & & 1.71538 & 2.78462 \\
\hline GrpD: $7^{\text {th }}$ day & 1 & 0.611012 & 1.38899 & & 0.465376 & 1.53462 \\
\hline GrpE: $1^{\text {st }}$ day & 2.25 & 1.68517 & 2.81483 & & 1.71538 & 2.78462 \\
\hline GrpE: $7^{\text {th }}$ day & 0.93 & 0.311882 & 1.54812 & & 0.395376 & 1.46462 \\
\hline
\end{tabular}

$P$-value (two-tailed). 


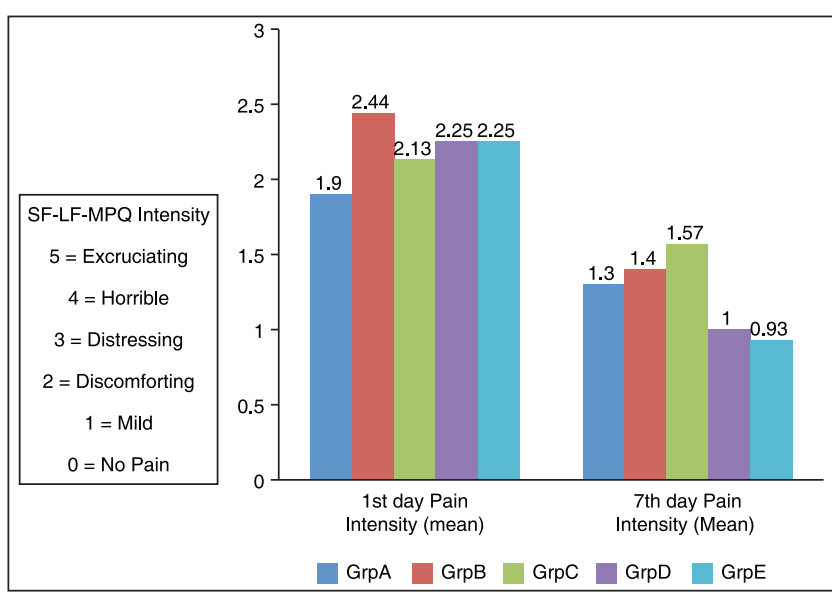

Figure 1: Intensity of Pain (mean) Before and after medication

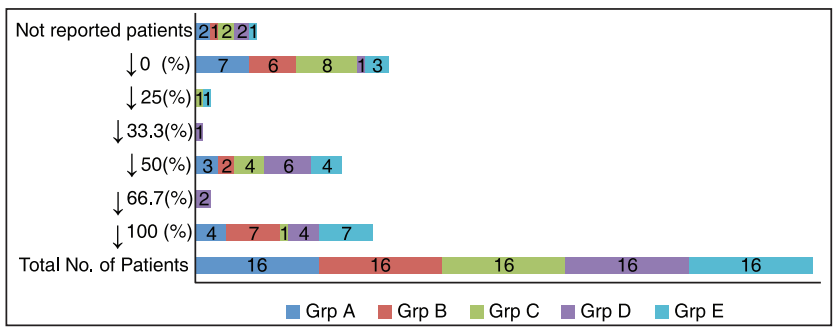

Figure 3: Number of patients reported to reduction of pain Intensity (Percent)

E respectively and insignificant, i.e. $(P>0.05)$ was 0.06603 for $\mathrm{Grp}$ C. So, comparatively pain reduction response was highly significant for the Group D $(P=0.0002027)$ than the GrpE $(P=0.002136)$ [Table 5].

The probability of pain reduction among Grp A (versus)Vs. D, Grp A Vs. E, Grp B Vs. D, Grp B Vs. E, Grp C Vs. D, Grp C Vs. E had 0.000175027, 0.00112602, 0.00117219, 0.00246735, $0.000432019,0.00111177$ respectively. Thus, it was indicating that the probability of the pain reduction was highly significant in Group D Vs. Group C $(P=0.00043)$, and Group D Vs. Group A $(P=0.00017)$ [Table 6].

\section{DISCUSSION}

Schug SA. 2006 reported in his study that, combination of analgesics acting by various mechanisms offer increased efficacy due to synergism/additive analgesic effects. So, the appropriate combinations when given increased analgesic efficacy and decreased adverse effects could be expected in comparison with either treatment alone. ${ }^{[6]}$ Aceclofenac is a proven effective NSAID, which is cox-2 selective, good GI tolerability and safer for cardiovascular system compared to other selective cox-2 inhibitors. ${ }^{[7]}$ Paracetamol has been considered as highly effective, i.e., first line drug for acute low back pain in all reviewed guidelines. Paracetamol is a good analgesic, antipyretic drug with weak anti-inflammatory effect. ${ }^{[8]}$ Tramadol is a centrally acting opioid analgesic produces analgesic effect that begins within one hour of its administration and reaches

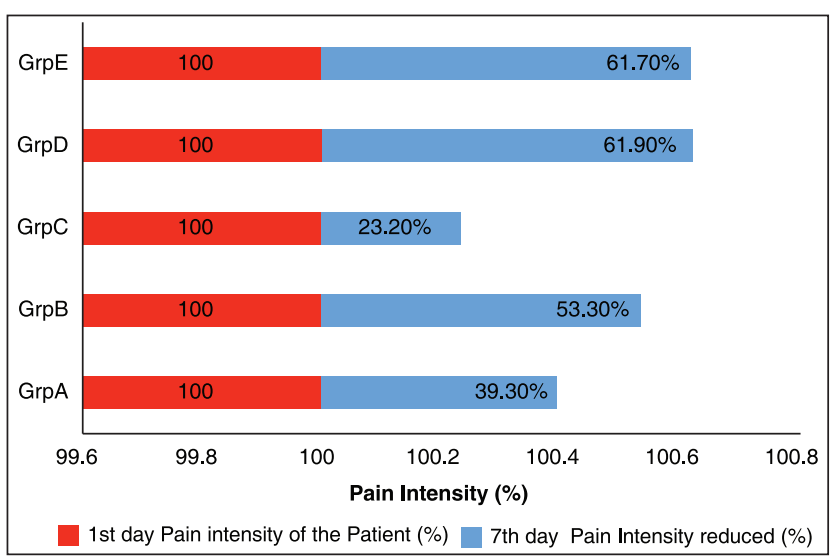

Figure 2: Intensity of Pain Reduction after medication (Percent)

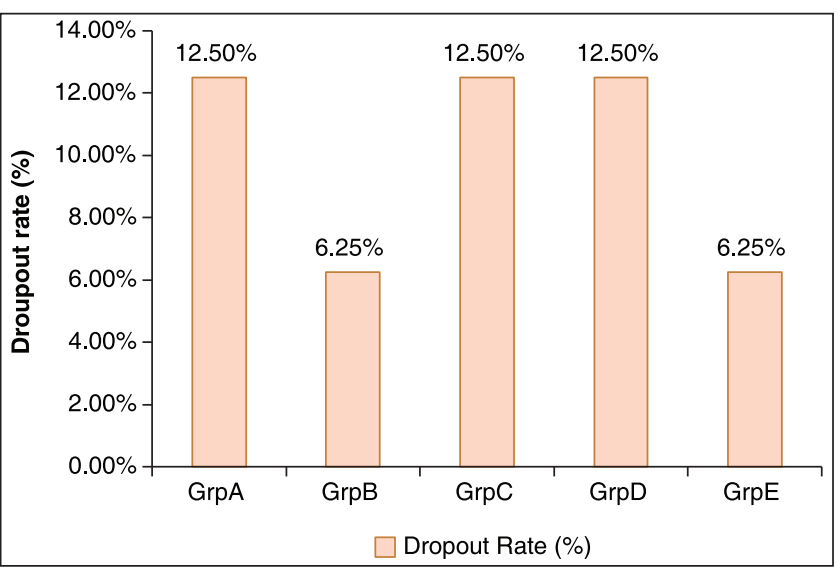

Figure 4: Number of patients not reported at end of the study (Dropout Rate \%)

a peak in 2 to 3 hours. Though, the oral opioids are effective in acute low back pain like sciatica, but it is not advisable for long term use. Thus, opioid should be considered a second- or third-line analgesic drug for a short period. ${ }^{[9]}$ Every living cell has a membrane potential (of about $-70 \mathrm{mV}$ ), if the membrane potential changes, it influences the movement of ions. The energy in the membrane (and other organelles of course) offers the potential to change the behaviour of the cell - one of the fundamental tenets of electrotherapy - and therefore make a difference to the behaviour of cells and tissues. Thus, Electrotherapy has a place within clinical practice. ${ }^{[10-12]}$ So, in the present study, the pain reduction response was highly significant for Aceclofenac $100 \mathrm{mg}$ + Paracetamol $500 \mathrm{mg}$ + IFT treated Grp $\mathrm{D}(P=0.0002027)$ and Tramadol $37.5 \mathrm{mg}+$ Paracetamol $325 \mathrm{mg}+$ IFT treated Grp E ( $P=0.002136)$ than compared to Group A, B, C treated medication, but among Group $\mathrm{D}$ and $\mathrm{E}$, the pain reduction was more in Group D than the Group E.

\section{LIMITATIONS}

This present study data has helped only viewing analgesic effectiveness of the Aceclofenac100 mg + Paracetamol $500 \mathrm{mg}$, Tramadol $37.5 \mathrm{mg}$ + Paracetamol $325 \mathrm{mg}$, Interferential therapy alone and combination with this study drugs. 


\begin{tabular}{|c|c|c|c|c|c|c|c|c|c|c|c|}
\hline Grps & $\begin{array}{l}\text { Before and } \\
\text { after therapy }\end{array}$ & $\begin{array}{c}\text { No. of } \\
\text { patients }\end{array}$ & $\begin{array}{c}\text { Pain intensity } \\
\text { (Mean) }\end{array}$ & S.D. & Result & $t$ statistics & df & $P$-value ${ }^{[1]}$ & $\begin{array}{c}\text { Mean } \\
\text { difference }\end{array}$ & $\begin{array}{l}\text { Lower } \\
\text { limit }\end{array}$ & $\begin{array}{c}\text { Upper } \\
\text { limit }\end{array}$ \\
\hline \multirow[t]{2}{*}{ Grp A } & 1st Day $^{\text {st }}$ & 16 & 1.9 & 0.5 & \multirow{2}{*}{$\begin{array}{l}\text { Equal } \\
\text { variance }\end{array}$} & \multirow{2}{*}{2.16392} & \multirow[t]{2}{*}{30} & \multirow{2}{*}{0.03856} & \multirow[t]{2}{*}{0.6} & \multirow[t]{2}{*}{0.0337328} & \multirow{2}{*}{1.16627} \\
\hline & $7^{\text {th }}$ Day & 16 & 1.3 & 0.99 & & & & & & & \\
\hline \multirow[t]{2}{*}{ Grp B } & $1^{\text {st }}$ Day & 16 & 2.44 & 1.09 & \multirow{2}{*}{$\begin{array}{l}\text { Equal } \\
\text { variance }\end{array}$} & \multirow[t]{2}{*}{2.19538} & \multirow[t]{2}{*}{30} & \multirow[t]{2}{*}{0.03601} & \multirow[t]{2}{*}{1.04} & \multirow[t]{2}{*}{0.0725362} & \multirow[t]{2}{*}{2.00746} \\
\hline & $7^{\text {th }}$ Day & 16 & 1.4 & 1.55 & & & & & & & \\
\hline \multirow[t]{2}{*}{ Grp C } & $1^{\text {st }}$ Day & 16 & 2.13 & 0.81 & \multirow{2}{*}{$\begin{array}{l}\text { Equal } \\
\text { variance }\end{array}$} & \multirow[t]{2}{*}{1.90778} & \multirow[t]{2}{*}{30} & \multirow[t]{2}{*}{0.06603} & \multirow[t]{2}{*}{0.56} & \multirow[t]{2}{*}{0.039474} & \multirow[t]{2}{*}{1.15947} \\
\hline & $7^{\text {th }}$ Day & 16 & 1.57 & 0.85 & & & & & & & \\
\hline \multirow[t]{2}{*}{ Grp D } & $1^{\text {st }}$ Day & 16 & 2.25 & 0.93 & \multirow{2}{*}{$\begin{array}{l}\text { Equal } \\
\text { variance }\end{array}$} & \multirow{2}{*}{4.2291} & \multirow[t]{2}{*}{30} & \multirow[t]{2}{*}{0.0002027} & \multirow[t]{2}{*}{1.25} & \multirow[t]{2}{*}{0.646366} & \multirow[t]{2}{*}{1.85363} \\
\hline & $7^{\text {th }}$ Day & 16 & 1 & 0.73 & & & & & & & \\
\hline \multirow[t]{2}{*}{ Grp E } & $1^{\text {st }}$ Day & 16 & 2.25 & 1.06 & \multirow{2}{*}{$\begin{array}{l}\text { Equal } \\
\text { variance }\end{array}$} & \multirow[t]{2}{*}{3.36013} & \multirow[t]{2}{*}{30} & \multirow[t]{2}{*}{0.002136} & \multirow[t]{2}{*}{1.32} & 0.517714 & 2.12229 \\
\hline & $7^{\text {th }}$ Day & 16 & 0.93 & 1.16 & & & & & & & \\
\hline
\end{tabular}

\begin{tabular}{|c|c|c|c|c|c|}
\hline Grps & $\begin{array}{l}\text { Before and } \\
\text { after therapy }\end{array}$ & $\begin{array}{c}\text { No. of } \\
\text { patients }\end{array}$ & $\begin{array}{l}\text { Pain intensity } \\
\text { (Mean) }\end{array}$ & S. D. & $\begin{array}{lccc}\begin{array}{l}\text { Source of sum of df } \\
\text { variation squares }\end{array} & \begin{array}{c}\text { Mean } \\
\text { square }\end{array} & \begin{array}{c}F \\
\text { statistics }\end{array} & P \text {-value } \\
\end{array}$ \\
\hline \multirow[t]{4}{*}{ Grp A Vs. Grp D } & $1^{\text {st }}$ Day & 16 & 1.9 & 0.5 & \multirow{4}{*}{$\begin{array}{l}\text { Between } \\
\text { Groups } 15.39,3,5.13,7.80852,0.000175027 \\
\text { Test for equality Chi square, df } \\
\text { of variance } 7.3049,3, P 0.0627892\end{array}$} \\
\hline & $7^{\text {th }}$ Day & 16 & 1.3 & 0.99 & \\
\hline & $1^{\text {st }}$ Day & 16 & 2.25 & 0.93 & \\
\hline & $7^{\text {th }}$ Day & 16 & 1 & 0.73 & \\
\hline \multirow[t]{4}{*}{ Grp A Vs. Grp E } & $1^{\text {st }}$ Day & 16 & 1.9 & 0.5 & \multirow{4}{*}{$\begin{array}{l}\text { Between } \\
\text { Groups } 16.8208,3,5.60693,6.0627,0.00112602 \\
\text { Test for equality Chi square, df } \\
\text { of variance } 9.93037,3, P 0.0191674\end{array}$} \\
\hline & $7^{\text {th }}$ Day & 16 & 1.3 & 0.99 & \\
\hline & $1^{\text {st }}$ Day & 16 & 2.25 & 1.06 & \\
\hline & $7^{\text {th }}$ Day & 16 & 0.93 & 1.16 & \\
\hline \multirow[t]{4}{*}{ Grp B Vs. Grp D } & $1^{\text {st }}$ Day & 16 & 2.44 & 1.09 & \multirow{4}{*}{$\begin{array}{l}\text { Between } \\
\text { Groups } 22.5452,3,7.51507,6.02603,0.00117219 \\
\text { Test for equality Chi square, df } \\
\text { of variance } 8.88783,3, P 0.03082\end{array}$} \\
\hline & $7^{\text {th }}$ Day & 16 & 1.4 & 1.55 & \\
\hline & $1^{\text {st }}$ Day & 16 & 2.25 & 0.93 & \\
\hline & $7^{\text {th }}$ Day & 16 & 1 & 0.73 & \\
\hline \multirow[t]{4}{*}{ Grp B Vs. Grp E } & $1^{\text {st }}$ Day & 16 & 2.44 & 1.09 & \multirow{4}{*}{$\begin{array}{l}\text { Between } \\
\text { Groups } 24.3344,3,8.11147,5.35428,0.00246735 \\
\text { Test for equality Chi square, df } \\
\text { of variance } 2.90851,3, P 0.405948\end{array}$} \\
\hline & $7^{\text {th }}$ Day & 16 & 1.4 & 1.55 & \\
\hline & $1^{\text {st }}$ Day & 16 & 2.25 & 1.06 & \\
\hline & $7^{\text {th }}$ Day & 16 & 0.93 & 1.16 & \\
\hline \multirow{4}{*}{$\begin{array}{l}\text { Grp C Vs. Grp } \\
\text { D }\end{array}$} & $1^{\text {st }}$ Day & 16 & 2.13 & 0.81 & \multirow{4}{*}{$\begin{array}{l}\text { Between } \\
\text { Groups } 15.8188,3,5.27293,6.94927,0.000432019 \\
\text { Test for equality Chi square, df } \\
\text { of variance } 2.26455,3, P 0.519346\end{array}$} \\
\hline & $7^{\text {th }}$ Day & 16 & 1.57 & 0.85 & \\
\hline & $1^{\text {st }}$ Day & 16 & 2.25 & 1.06 & \\
\hline & $7^{\text {th }}$ Day & 16 & 1 & 0.73 & \\
\hline \multirow[t]{4}{*}{ Grp C Vs. Grp E } & $1^{\text {st }}$ Day & 16 & 2.13 & 0.81 & Between \\
\hline & $7^{\text {th }}$ Day & 16 & 1.57 & 0.85 & Groups17.5296, 3, 5.8432, 6.07433, 0.00111177 \\
\hline & $1^{\text {st }}$ Day & 16 & 2.25 & 1.06 & Test for equality Chi square, df \\
\hline & $7^{\text {th }}$ Day & 16 & 0.93 & 1.16 & of variance $2.59683,3, P 0.458046$ \\
\hline
\end{tabular}

\section{CONCLUSION}

According to the finding and within the limitation of the present study, the combination of IFT $(25-100 \mathrm{~Hz})$ application $15 \mathrm{~min}$ at Lumbar region daily for 7 days + Tablet (Aceclofenac $100 \mathrm{mg}+$ Paracetamol $500 \mathrm{mg}$ ) 1BD daily for 7 days is good combination as an analgesics, than, the Tablet (Aceclofenac $100 \mathrm{mg}+$ Paracetamol $500 \mathrm{mg}$ ) alone. The combination of IFT application + Tablet (Tramadol $37.5 \mathrm{mg}+$ Paracetamol $325 \mathrm{mg}$ ) 1 BD daily for 7 days is good combination analgesics, than, the Tablet (Tramadol $37.5 \mathrm{mg}+$ Paracetamol $325 \mathrm{mg}$ ) alone. But, the IFT + (Aceclofenac $100 \mathrm{mg}+$ Paracetamol $500 \mathrm{mg}$ ) therapy relieved pain much better, than IFT + (Tramadol $37.5 \mathrm{mg}+$ Paracetamol $325 \mathrm{mg})$. So, Tramadol $37.5 \mathrm{mg}+$ Paracetamol $325 \mathrm{mg}$ + IFT (25-100 Hz) should be given $2^{\text {nd }}$ or $3^{\text {rd }}$ preference (van Tulder MW, 2006 also reported that tramadol should be $2^{\text {nd }}$ or $3^{\text {rd }}$ line drug) ${ }^{[0]}$ than the (Aceclofenac $100 \mathrm{mg}+$ Paracetamol $500 \mathrm{mg}$ ) + IFT. The Group A, B, C measures can also be used for the relief the pain, but the analgesic response was lesser than the group D, E measures application.

\section{ACKNOWLEDGEMENTS}

We acknowledge the guidance of Dr. Shafee Mohd. Professor and Head, Dept. of Community Medicine, who given valuable guidance for 
the statistical calculations. I acknowledge pharmacist, Department of Physiotherapy, Orthopedics, Pharmacology and DSMCH management for the providing the valuable support.

\section{REFERENCES}

1. Blenkinsopp A, Paxton P, Blenkinsopp J. Symptoms in the pharmacy: A guide to the management of common illness. $6^{\text {th }}$ ed. Hoboken, New Jersey: Wiley Blackwell; 2009.

2. Doherty M, Lanyon P, Ralston SH. Presenting problems in musculoskeletal disease; Back and Neck pain. In: Boon NA, Colledge NR, Walker BR, editors. Davidson's The principle and Practice of Medicine. $20^{\text {th }}$ ed. Churchill Livingstone, London: Elsevier; 2006. p. 1083-4.

3. Coste J, Delecoeuillerie G, Cohen de Lara A, Le Parc JM, Paolaggi JB. Clinical course and prognosis factors in acute low back pain: An inception cohort study in primary care practice. Br Med J 1994;308:577-80.

4. Watson T, editor. Electrotherapy: Evidence Based Practice. Edinburgh: Churchill Livingstone, Elsevier; 2008.

5. Melzack R. The McGill Pain Questionnaire: Major properties and scoring methods. Pain 1975;1:277-99.

6. Stein C, Mendl G. The German counterpart to Mc-Gill Pain Questionnaire. Pain 1988;32:251-5.
7. Schug SA. Combination analgesia in 2005: A rational approach: Focus on paracetamol tramadol. Clin Rheumatol 2006;25:S16-21.

8. The COX-2 inhibitor etoricoxib did not alter the anti-platelet of low dose aspirin in health in healthy volunteers. Arthritis Rheum 2001;44 (Suppl 9):S135.

9. Coste J, Delecoeuillerie G, Cohen de Lara A, Le Parc JM, Paolaggi JB. Clinical course and prognosis factors in acute low back pain: An inception cohort study in primary care practice. Br Med J 1994;308:577-80.

10. Van Tulder MW, Scholten RJ, Koes BW, Deyo RA. Nonsteroidal antiinflammatory drugs for low back pain. Cochrane Database Syst Rev 2006;2:CD000396.

11. Watson T. Electrical Stimulation for Wound Healing: A Review of Current Knowledge. In: Electrotherapy — Evidence Based Practice. In: Kitchen S, editor. London: Churchill Livingstone; 2002.

12. Veenman $P$, Watson $T$. A physiotherapy perspective on pain management UK Vet 2005;10:87-91.

How to cite this article: Vidyarthi SK, Nayak RP, Krishna S, Selvaraj P, Gupta SK, Kumar BA. "Low back pain response after application of interferential therapy alone and in combination with aceclofenac+paracetamol, tramadol+paracetamol: A prospective, comparative, clinical study". Int J Med Public Health 2014;4:231-6.

Source of Support: DSMCH, Conflict of Interest: Nil. 